\title{
Potential limitations of the Sleeping Beauty transposon use in gene expression studies*
}

\author{
Weronika Sowińska, Mateusz Wawro, Aleksandra Solecka and Aneta Kasza ${ }^{\varpi}$ \\ Department of Cell Biochemistry, Faculty of Biochemistry, Biophysics and Biotechnology, Jagiellonian University, Kraków, Poland
}

\begin{abstract}
MCPIP2 is the least known member of the MCPIP family of proteins. Recently we have found that it is a new RNase involved in transcript turnover. However, the full spectrum of its cellular targets is still unidentified. To discover transcripts which are regulated by this protein we have employed Sleeping Beauty transposons. This tool allows for rapid generation of a stable transgenic cell line with inducible expression of the desired gene. In this study, we analysed how the Sleeping Beauty system itself influences expression of chosen genes, namely IL6 , Regnase- 1 and VEGF. We found that the system alone may influence expression of IL- 6 . Our results indicate that Sleeping Beauty transposons should be used with caution in studies that are focused on changes in the transcript level.
\end{abstract}

Key words: transcriptome analysis, Sleeping Beauty transposons, inducible gene expression

Received: 31 March, 2019; revised: 28 May, 2019; accepted: 13 June, 2019; available on-line: 12 July, 2019

凶e-mail: aneta.kasza@uj.edu.p

Acknowledgments of Financial Support: This work was funded by the National Science Centre, Poland (NCN, OPUS8 Funding scheme, project number UMO-2014/15/B/NZ2/03379 to A.K.). Faculty of Biochemistry, Biophysics, and Biotechnology of Jagiellonian University is a partner of the Leading National Research Center (KNOW) supported by the Ministry of Science and Higher Education.

${ }^{*}$ A preliminary report on this subject was presented at the 46th Winter School of the Faculty of Biochemistry, Biophysics and Biotechnology, Jagiellonian University in Krakow, Zakopane, Poland, February 11th-15th 2019

Abbreviations: AAV, adeno-associated virus; CGAS, cyclic GMP-AMP synthase; CRISPR, Clustered Regularly Interspaced Short Palindromic Repeats; DAI, DNA-dependent activator of interferon-regulatory factors; Dox, doxycycline; DMEM, Dulbecco's Modified Eagle Medium; dsRNA, double stranded RNA; GOI, gene of interest; GFP, green fluorescent protein; gRNA, guide RNA; HDR, homologous recombination; IL-6, Interleukin 6; ITR, inverted terminal repeats; Luc, luciferase; MCPIP, monocyte chemoattractant protein-induced protein; miRNA, micro RNA; mRNA, messenger RNA; NHEJ, nonhomologous end joining; PCR, polymerase chain reaction; Reg-1, Regnase 1; RNAseq, RNA sequencing; SB system, Sleeping Beauty system; sgRNA, single guide RNA; siRNA, silencing RNA; TALEN, Transcription Activator-Like Effector Nuclease; TRE, tetracycline response element; VEGF, vascular endothelial growth factor

\section{INTRODUCTION}

Capability to modulate expression of a specific gene has been beneficial to understand its function. Since discovery of the RNA interference phenomenon in 1998, double-stranded RNAs (dsRNAs), such as long dsRNAs, siRNAs, and microRNAs, have been used as research tools to down-regulate an mRNA level by its cleavage, degradation or translational repression (the last one mainly through miRNA) (Lam et al., 2015). However, delivery of exogenous dsRNAs in mammalian cells can be associated with unintentional activation of pattern recognition receptors of the innate immune system and induction of the interferon pathway (Gantier \& Williams, 2007). As an effect, unwanted changes in the level of different genes' induction are observed and can be the source of false conclusions. Recently, new tools for genome editing, such as Zinc Finger Nucleases, TALENs (Transcription Activator-Like Effector Nuclease) and CRISPR (Clustered Regularly Interspaced Short Palindromic Repeats)-Cas have emerged (Gaj et al., 2013). The first two employed complicated protein-DNA interactions for targeting the gene of interest (GOI), while the CRISPR-Cas system uses small RNAs with an on-target specificity. All three systems generate double-stranded DNA breaks which can be repaired through non-homologousend joining (NHEJ) or homologous recombination (HDR). In the course of NHEJ, insertions or deletions can be created which in turn may result in frame-shift mutations and generation of premature STOP codons. HDR-mediated repair is more predictable than NHEJ, however, it occurs with lower frequency and additionaly a template for recombination has to be delivered into the cell (Liu et al., 2019). The biggest threat concerning the use of the known tools for genome editing is their potential to induce unwanted, off-target effects. This disadvantage also applies to RNAi technology.

An opposite approach for studying a gene's function is its overexpression. Two types of vectors are the most frequently used for efficient delivery of transgenes, non-viral (plasmids) and viral vectors. When plasmids are employed as a delivery vehicles, transcription of the transgene proceeds with an efficiency depending on the type of the promoter present in the plasmid. Viral promoters orchestrate expression of a transgene at a very high level which quickly diminishes over several days. Use of eukaryotic promoters results in longer and moderate expression of a given transgene. Maintaining prolonged selection pressure can lead to integration of the expression vector into the genome. However, this is a rare event and prone to artifacts (Zucca et al., 2013). DNA delivery to mammalian cells can be also propagated by viral vectors derived from retroviruses, adenoviruses, adeno-associated viruses (AAVs), herpesvirus and poxvirus. Retroviral vectors can stably integrate into the genome of the infected cell but require cell division for transduction. Cell division is not needed during infection with adenoviruses and herpes virus but there is no integration into the host genome and the expression of the transgene is transient. AAVs also infect many nondividing and dividing cell types but have a limited DNA capacity $(4 \mathrm{~kb})$. Poxvirus vectors have high capacity but exert potential cytopathic effects (Kotterman et al., 2015; Walther \& Stein, 2000). 
Most of the known plasmid vectors suffer from the limited duration of transgene expression. Lack of genomic insertion causes cellular degradation and/or dilution of the vector. An additional problem concerns the cellular toxicity of the transfection process. This is mainly related to the high concentration of recombinant proteins which results in activation or overloading of specific biological pathways or in aggregate creation of recombinant proteins (Vavouri et al., 2009). Therefore, despite the fact that cell transfection had been carried out since the 1990s, the need for better tools for transgene overexpression is still persisting.

The potential of transposable elements for genome modification has been analyzed since the nineties. These natural elements have the ability to move within the genome through a cut-and-paste mechanism. The DNA transposons contain a transposase gene flanked by inverted terminal repeats (ITRs) which carry the transposase binding sites. Transposase catalyzes excision of the transposon from its original location and promotes its translocation. Importantly, it is possible to separate both functional components of transposons. Any GOI flanked by ITRs can undergo transposition in the presence of a transposase supplied in trans. The synthetic Sleeping Beauty transposon is an equivalent to an ancient $\mathrm{Tc} 1 /$ mariner-like element present in the salmonid fish genome. Given that this element was inactive for 10 million years and then reactivated in 1997, it was named Sleeping Beauty (SB), after the famous fairy tale. The SB has been generated by "reverse engineering" from defective copies of an ancestral transposon in fish. Its reactivation included homology comparison in combination with site-directed mutagenesis. Additionally, its transpositional activity was enhanced by obtaining a hyperactive mutant of SB transposase, named SB100x (Kowarz et al., 2015; Kebriaei et al., 2017; Hudecek et al., 2017). Advantages of the SB system include permanent genomic insertion and the ability to maintain and propagate transposon vectors as plasmid DNA, meaning simple and inexpensive manufacture. Another advantage of the SB system is the target site specificity. Integration of SB transposons occurs at sequences containing TA-dinucleotides. There are about 30000 such sequences in the human genome. This is an important improvement of SB system over viral vectors which integrate into the genome at promoters and first introns of actively transcribed genes. The SB system is also better for gene delivery than viral vectors in terms of its lower immunity which is a significant factor for in vivo studies (Kowarz et al., 2015).

Previously, we have found that MCPIP2 is involved in regulation of inflammation through destabilization of transcripts of proinflammatory cytokines (Wawro et al., 2019) To identify new RNA molecules which are regulated by MCPIP2, we employed the SB system for overexpression of MCPIP2 in a human astrocytoma cell line, U251-MG. Surprisingly, we found that the SB system alone influences expression of some genes. The aim of this work was to analyse the use of the Sleeping Beauty tool for gene expression studies.

\section{MATERIALS AND METHODS}

Cell culture. Human astrocytoma U251-MG (ECACC 09063001), formely known as U373-MG cell line, was used in experiments. It was derived from a 75-year old male patient with malignant glioblastoma multiforme tumour by an explant technique, and established at the Wallenberg laboratory, Uppsala, Sweden (Westermark et al., 1973). It was cultured in Dulbecco's Modified Eagle Medium (DMEM) with $4.5 \mathrm{~g} / \mathrm{L}$ D-glucose (BioWest, Nuaillé) supplemented with $10 \%$ (v/v) Fetal Bovine Serum Tetracycline free (BioWest, Nuaillé), at $37^{\circ} \mathrm{C}$ in a humified atmosphere with $5 \% \mathrm{CO}_{2}$.

Construct preparation. The pSBtet-GP-MCPIP2 construct was prepared by cloning $\mathrm{ZC} 3 \mathrm{H} 12 \mathrm{~B}$ coding region (gene encoding MCPIP2, NM_001010888.3) into the Sleeping Beauty vector pSBtet-GP (Addgene, \#60495). PCR products were amplified from a previously prepared vector pcDNA5/FRT/TO-SF-ZC3H12B, containing ZC3H12B coding region fused with StrepTagII and FLAG tags using Q5 High Fidelity DNA Polymerase (New England Biolabs, Ipswich) (Wawro et al., 2019). The following primers were used:

forward: AGGCCTCTGAGGCCACCCACCATGGCAAGCTGGAGC,

reverse: AGGCCTGACAGGCCTCAACGTGCAGCCCTAAGC

After gel electrophoresis, PCR products were excised, purified (Gel/PCR ME Mini Kit, Syngen, Wroclaw), cleaved with the SfiI restriction enzyme (New England Biolabs, Ipswich), and purified after enzymatic reaction. Then, products were ligated into gel-purified pSBtet-GP vector linearized with the SfiI enzyme by using a T4 DNA ligase (Thermo Fisher Scientific, Waltham). Obtained constructs were verified by restriction analysis and sequencing.

Transfection and cell selection. U251-MG cells were seeded in 12-well plates at a density of $1 \times 10^{5}$ cells/ well. 24 hours later, the cells were transfected with the pSBtetGP-MCPIP2 vector and the pCMV(CAT) T7-SB100 vector (Addgene, \#34879), using the TransIT-LT1 Transfection Reagent (Mirus, Madison) according to the manufacturers' instructions. The total amount of $1 \mu \mathrm{g}$ of DNA per well was used in a ratio of 9:1 (pSBtet-GP: pCMV(CAT)T7-SB100). The next day, the cells were transferred to a 6 -well plate and puromycine (Invivogen, San Diego) was added to a final concentration of $1 \mu \mathrm{g} / \mathrm{ml}$. After a week of selection, stable cell line was generated. Transfection efficiency was assessed by observation of GFP fluorescence under a microscope.

RNA isolation and cDNA preparation. Total RNA was isolated from U251-MG cells modified with the Sleeping Beauty system using the Chomczynski method (Chomczynski \& Sacchi, 2006). The RNA concentration was measured using NanoDrop ND-1000 spectrophotometer (Thermo Fisher Scientific, Waltham). $1 \mu \mathrm{g}$ of RNA was treated with RQ1 RNase-free DNase (Promega, Madison) according to the manufacturer's instructions, and then reverse-transcribed to cDNA with M-MLV-Reverse transcriptase (Promega, Madison) and $500 \mathrm{ng}$ of oligo(dT) $)_{15}$ primers (Genomed, Warsaw) according to the manufacturer's instructions.

Real-time PCR. Real-time PCR was performed using SYBR-A RT HS-PCR Mix (A\&A Biotechnology, Gdynia) and primers specific to analyzed transcripts (Genomed, Warsaw):

\footnotetext{
IL-6: forward: GACAGCCACTCACCTCTTCA reverse: AGTGCCTCTTTGCTGCTTTC

Regnase-1: forward: GGAAGCAGCCGTGTCCCTATG, reverse: TCCAGGCTGCACTGCTCACTC

VEGF: forward: ATGCGGATCAAACCTCACCAAGGC reverse: TTAACTCAAGCTGCCTCGCCTTGC

EF-2: forward: GGTGCAGTGCATCATCGAGGAGTC, reverse: TCGCGGTACGAGACGACCGG
} 
mRNA level in each sample was analyzed in duplicate, and the results were normalized to the reference gene (EF-2). The relative level of transcripts was calculated by using the $\Delta \Delta \mathrm{C}_{\mathrm{T}}$ method.

Western Blot analysis. U251-MG cells with inducible overexpression of MCPIP2 were plated in a 12-well plate. The following day, protein expression was induced by adding doxycycline to a final concentration of $1 \mu \mathrm{g} /$ ml. 24-h after induction, induced cells as well as non-induced control cells were lysed in $100 \mu \mathrm{l} /$ well of Laemmli lysis buffer $(0.35 \mathrm{M}$ Tris $\cdot \mathrm{HCl}, 35 \%$ (v/v) glycerol, $10 \%$ (w/v) SDS, $3.6 \mathrm{M} \beta$-mercaptoethanol, $0.12 \mathrm{~g} / \mathrm{ml}$ bromophenol blue) and denaturated at $95^{\circ} \mathrm{C}$ for $7 \mathrm{~min}-$ utes. $20 \mu \mathrm{l}$ of samples were separated by SDS-PAGE and wet transfer was performed onto PVDF membrane (Merc Millipore, Burlington). After transfer, the membranes were blocked in $5 \%(\mathrm{w} / \mathrm{v})$ non-fat dry milk in TTBS (20 mM Tris, $150 \mathrm{mM} \mathrm{NaCl,} \mathrm{0,1 \%} \mathrm{Tween-20),}$ transferred to an antibody solution, and incubated overnight at $4^{\circ} \mathrm{C}$ with gentle agitation. The following primary antibodies were used: anti-FLAG (D6W5B, 1:2000 Cell Signaling, Danvers) or anti- $\beta$-actin (13E5, 1:2000 Cell Signaling, Danvers). After primary antibody incubation, the membranes were washed three times for 5 minutes at room temperature with TTBS, and then incubated with HRP-conjugated secondary antibodies (anti-rabbit, 1:10000, Cell Signalling, Danvers) for 1 hour at room temperature with gentle agitation. Then, the membranes were washed three times with TTBS (as previously), incubated with Clarity Western ECL Blotting Substrate (BioRad, Hercules) for 5 minutes at room temperature, and chemiluminescence was detected using Fusion-Fx system (VilberLourmat, Marne-la-Vallée).

Generation of a knockout cell line and analysis of mutations. U251-MG cells were modified using CRISPR/Cas9 method targeting the ZC3H12B gene to generate a cell line without MCPIP2 expression (U251MG-M2-KO). Cas9 mRNA was transcribed in vitro using the px330 plasmid (Addgene \#42230) linearized with $\mathrm{XbaI}$ (New England Biolabs, Ipswich) as a template, according to standard mRNA synthesis protocol (New England Biolabs, Ipswich, E2060). Next, cells were transfected with synthesized Cas9 mRNA together with sgRNA targeting ZC3H12B (CRISPR961504_SGM, Thermo Fisher Scientific, Waltham) in 4 hour interval using Messenger Max and RNAiMAX (Thermo Fisher Scientific, Waltham), respectively, according to the manufacturer's instructions. After transfections, the cells were seeded in 96-well plates at a 0.5 cell/well density to obtain single cell clones. Presence of a mutation in the desired gene was analyzed using the mismatch sensitive nuclease CelI (Qiu et al., 2004). Genomic DNA was isolated from the obtained cell clones and wild type cells by lysis in Proteinase $\mathrm{K}$ lysis buffer $(50 \mathrm{mM}$ Tris-HCl, $\mathrm{pH}$ 8.0, $1 \mathrm{mM}$ EDTA, 0.5\% Triton-X supplemented with $1 \mu \mathrm{g} / \mu \mathrm{l}$ of Proteinase $\mathrm{K}$ ) and PCR was performed using primers flanking the targeted DNA region. The following primers were used: forward: CCGCATGTGCT'T'TCAGGAG, reverse: ACCAGGCTTACCTCATTGCC. PCR products were reannealed by heating to $95^{\circ} \mathrm{C}$ and gradual cooling to $4^{\circ} \mathrm{C}$. In addition, reannealing of each clone with products obtained with gDNA from wild type cells was performed. In the next step, two reactions were prepared for each tested clone: by mixing PCR product itself $(4 \mu \mathrm{l})$, or by mixing PCR product $(2 \mu \mathrm{l})$ with the product obtained with wild type gDNA $(2 \mu \mathrm{l})$. To each reaction, $6 \mu \mathrm{l}$ of $1 \times$ Cell buffer was added. Heteroduplexes were cleaved by adding $0.5 \mu \mathrm{l} /$ reaction of the mismatch sensitive nuclease CelI that recognizes single, unpaired nucleotides and performs cleavage. CelI and CelI buffer were prepared as described by Till and others (Till et al., 2006). Products of cleavage were separated in $1 \%$ agarose gel $(\mathrm{w} / \mathrm{v})$ in $1 \times \mathrm{TAE}$ buffer supplemented with $5 \mu \mathrm{g} / \mathrm{ml}$ of ethidium bromide and presence of the mutation was analyzed. Presence of mutation in selected cloneswas also confirmed by sequencing (Genomed, Warsaw).

Statistical analysis and graphs. Statistical analysis and graphs were performed using GraphPad Prism (v. 5.0, GraphPad Software Inc.). Scheme of expression cassettes was obtained by using Adobe Illustrator CC (2019). Detailed information about statistical tests is indicated in figure descriptions.

\section{RESULTS AND DISCUSSION}

\section{Generation of a U251-MG cell line with MCPIP2 knockout}

To maximize the difference between transcriptomes from cells with overexpression of MCPIP2 and control (reference) cells, we have generated a U251-MG cell line with MCPIP2 gene knockout (U251-MG-M2-KO cells) by the CRISPR-Cas9 system. We decided to use the allRNA approach in which Cas9 and chemically modified gRNA are delivered into cells in an RNA form. In comparison with unmodified gRNA, synthetic gRNA shows improvement in stability and increased efficiency of genome editing when Cas9 mRNA is co-delivered into the cells (Hendel et al., 2015). This approach is also less toxic than the classic approach. In cells transfected with plasmid DNA the Cas9 protein accumulates over time, whereas in Cas9 mRNA transfected cells the protein level is relatively low with the greatest peak four hours posttransfection. Because of this features, off-target activity for the all-RNA approach is lower than that of plasmid DNA transfection (Ranganathan et al., 2015). Using the all-RNA approach we were able to obtain a stable cell line with $Z C 3 H 12 B$ gene editing, confirmed by the CelI nuclease assay and sequencing (Fig. 1). After transfection with Cas9 mRNA and sgRNA, the cells were seeded into a 96-well plate to obtain single cell clones. The clones were tested using the CelI mismatch sensitive nuclease to discriminate whether a mutation aroused, as well as to determinate if one, or both gene copies were altered (Fig. 1A). To do that, PCR was performed with DNA obtained from mutants as well as with wild type DNA. Then, mutant PCR products were reannealed, and additional reaction was performed at the same time for each clone by annealing equal amounts of mutant and wild type PCR products. After reannealing, heteroduplexes were subjected to CelI cleavage, and products were visualized by gel electrophoresis. In case of heteroduplexes from mutant PCR, if cleavage products are present, a mutation occurred only in one allele (heterozygote, $+/-$ ). However, absence of cleaved products can represent two situations: no mutation (wild-type, $+/+$ ) or mutation present in both alleles (homozygote, $-/-$ ). To discriminate between these two options, additional annealing reaction with wild-type PCR is necessary. In this situation, if cleavage products are present we can assume that the mutation occurred in both gene copies (Fig. 1A). In our case, we obtained three clones that harbor mutation in both alleles (K5, K7, K12, Fig. 1B). Sequencing revealed that only in the genome of clone K5 the CRISPR/Cas9 cleavage caused a deletion of a single nucleotide that leads to a frame-shift mutation and generation of a pre- 


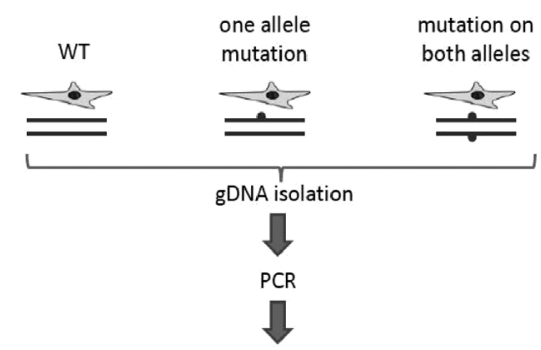

reannealing of PCR products

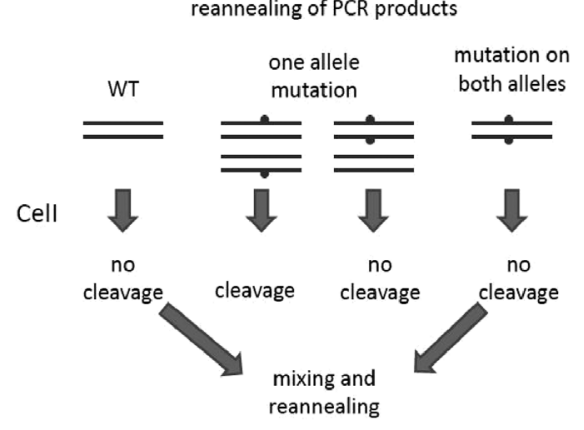

B
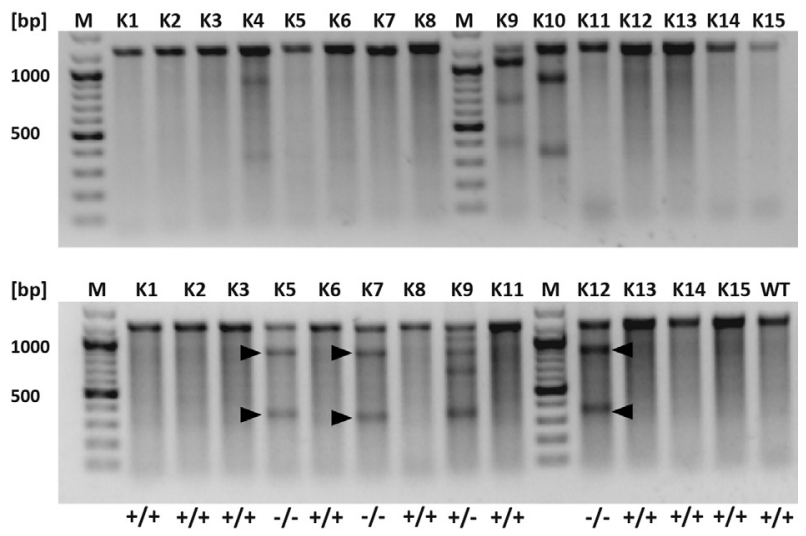

C

WT GAGCTCTGAGAGTGACCCTGAACAGATAAGCCTTAAGAGTAGCGACAACAGCAAGAGCTG 811 K5 GAGCTCTGAGAGTGACCCTGAACAGATAAGCCTTAAGAGTAGCGACAACAGCAAGAGCTG 838 K7 GAGCTCTGAGAGTGACCCTGAACAGATAAGCCTTAAGAGTAGCGACAACAGCAAGAGCTG 838 K12 ------------

WT CCAACCTAGGGATGGTCAGTTGAAGAAAAAGGAGATGCACTCCAAGCCACACCGCCAGCT 871 K5 CCAACCTAGGGATGGTCAGTTGAAGAAAAAGGAGATGCACTCCAAGCCACACCGCCAGCT 898 K7 CCAACCTAGGGATGGTCAGTTGAAGAAAAAGGAGATGCACTCCAAGCCACACCGCCAGCT 898 K12 $\nabla$

WT CTGTCGATCACCCTGCCTAGATCGTCCAAGTITCTCCCAGAGCAGCATTTTACAGGATGG 931 K5 CTGTCGATCACCCTGCCA-RATCGTCCAAGTTTCTCCCAGAGCAGCATTTTACAGGATGG 957 K7 CTGTC---------GTCCAAGTTTCTCCCARAGCAGCATTTTACAGGATGG 940 K12 CTGTCGATCACC------ CAAGTTTCTCCCAGAGCAGCATTTTACAGGATGG 774

Figure 1. Analysis of mutation in the $Z C 3 H 12 B$ gene with Cell mismatch sensitive nuclease.

(A) Schematic representation of mutation analysis using the Cell mismatch sensitive nuclease. (B) Analysis of mutants with the use of Cell nuclease. PCR was performed with gDNA isolated from mutants (K1-K15), as well as wild type U251-MG cells (WT). Mutant PCR products and mixtures of mutant and wild type PCR products were reannealed, and cleavage with the Cell nuclease was performed. Upper panel: cleavage of mutant PCR products after reannealing. Lower panel: cleavage of heteroduplexes of WT PCR product and PCR products from the upper panel. K1-K15, analysed clones. Arrows on the lower panel indicate products of Cell cleavage. "-/-"," +/-", "+/+" indicate homozygote, heterozygote or wild type genotype, respectively. (C) Alignment of wild type U251-MG (WT) and K5, K7, K12 mutant DNA fragments after CRISPR/Cas9 genome editing targeting $Z C 3 H 12 B$. Image presents fragment of the $Z C 3 H 12 B$ sequence where mutation occurs. "-" indicates deleted nucleotides. Black arrow indicates the position of nucleotide deleted in $\mathrm{K} 5$, leading to a frame-shift.
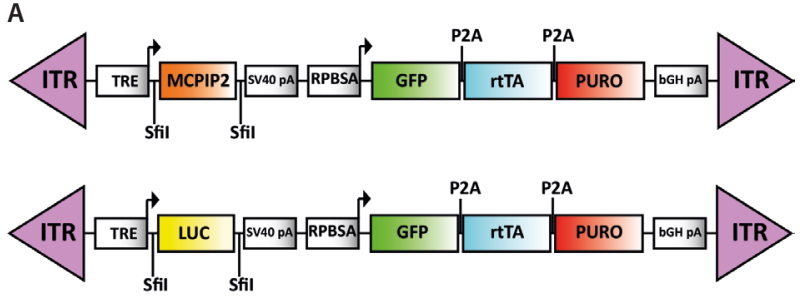

B


Figure 2. Generation of U251-MG cell line with the Sleeping Beauty transposon-based inducible overexpression of MCPIP2 protein.

(A) Backbone of pSBtet-GP and pSBtet-GP-MCPIP2 vectors used for generation of the U251-MG-LUC and U251-MG-M2-SB cell lines, respectively. ITR, inverted terminal repeats; TRE, inducible promoter with tetracycline response element; MCPIP2, coding sequence for MCPIP2 with StrepTagll and Flag tags; LUC, coding sequence for luciferase. SV40 pA, polyadenylation signal; RPBSA, constitutive promoter; GFP, Green Fluorescence Protein reporter gene; rtTA, reverse Tetracycline transactivator gene; PURO, puromycine selection gene; bGHpA, polyadenylation signal (Modified from Kowarz et al., 2015). (B) Representative images of U251-MGM2-KO cells transfected with the pSBtet-GP-MCPIP2 vector 24 hours after transfection in phase contrast and fluorescence (GFP).

mature termination codon (Fig. 1C). Thus, clone K5 was used for further experiments and was modified by the Sleeping Beauty system.

\section{Generation of a U251-MG cell line with inducible overexpression of MCPIP2 protein}

MCPIP2 is the least known member of the MCPIP protein family which participates in regulation of inflammation through degradation of inflammatory transcripts and modification of cell signaling pathways. Our data indicate that it possesses an RNase activity, however, its molecular targets are largely unknown (Wawro et al., 2019). Our aim was to identify RNA species regulated by MCPIP2. In order to do that, we developed a stable U251-MG cell line with inducible overexpression of MCPIP2 using the SB transposon system. The genetic construct was prepared on the basis of the pSBtet-GP vector (Fig. 2A). This vector contains an expression cassette encoding MCPIP2 flanked by SB ITRs which allows its integration into the genome in the presence of the SB transoposase, and results in generation of a stable cell line with inducible overexpression of MCPIP2 (pSBtet-GP-MCPIP2). Expression cassette in pSBtetGP contains inducible and constitutive promoters that control transcription of different genes. A constitutive promoter (RPBSA) drives expression of the reporter protein (GFP), the selection marker (puromycin resistance gene) and rtTA protein. The last one in the presence of doxycycline(Dox) is capable of binding to the TRE (tetracycline response element) site that is present within the inducible promoter. This binding initializes expression of the downstream expression cassette (MCPIP2 or luciferase). Integration of the expression cassette into the genome is possible in the presence of a transposase, which is encoded by a separate vector called 




B

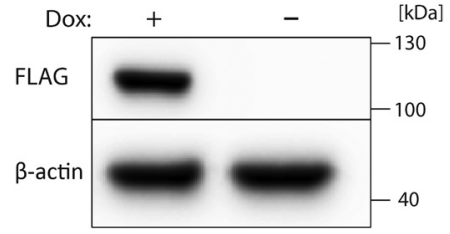

Figure 3. Analysis of MCPIP2 expression in the U251-MG-M2-SB cell line.

(A) MCPIP2 mRNA level in U251-MG-M2-SB (M2-SB) and U251-MGLUC (LUC) cells. Cells were treated with doxycycline (Dox+) or left untreated (Dox-) for 24 hours, and the level of MCPIP2 mRNA was measured by real-time PCR. The plot shows mean results from three independent experiments $(n=3) \pm S$.D., $* * * p<0.001$. Two-way ANOVA with Bonferroni's post-hoc test was used. (B) MCPIP2 expression in U251-MG-M2-SB (M2-SB) cells. Cells were treated with doxycycline (Dox+) for 24 hoursor left untreated (Dox-) and MCPIP2 protein level was examined by Western Blot analysis with anti-FLAG antibody. $\beta$-actin was used as a loading control (lower panel).

pCMV(CAT)T7-SB100 (pCMV-SB100X). Both vectors have to be co-delivered into the cells during transfection in the right proportions. A higher amount of vector encoding transposase results in more copies of transgene being integrated into the genome. Thanks to the presence of the selection marker and reporter GFP protein, efficiency of transfection can be assessed by simple observation of GFP fluorescence under a microscope, and non-transfected cells can be eliminated by the presence of puromycin (Fig. 2B). To avoid different genetic backgrounds, modified cell line (U251-MG-M2-KO) was used for genome editing by the SB transposon system. Within a week we were able to obtain a stable cell line with inducible overexpression of the MCPIP2 protein (U251-MG-M2-SB cell line), which was confirmed by real-time PCR and Western Blot analysis (Fig. 3A, B). In parallel, we have also generated a stable cell line with inducible overexpression of luciferase, which was obtained by transfection of U251-MG-M2-KO cells with the pSBtet-GP vector (U251-MG-LUC).

Sleeping Beauty system itself influences changes in the level of transcripts that are known MCPIP2 targets

Using U251-MG-M2-SB cells with inducible overexpression of MCPIP2 and U251-MG-M2-KO cells with MCPIP2 gene knockout, we decided to examine the level of transcripts that are known to be MCPIP2 targets. We also examined the level of targets which are not regulated by this RNase (Wawro et al., 2019). As a negative control, we have used the U251-MG-LUC cell line with inducible expression of luciferase. Recently, we have shown that IL-6 mRNA possessa stem-loop structure in its 3'UTR which is recognized by MCPIP2 and is involved in the interaction between MCPIP2 and this transcript, followed by mRNA degradation (Wawro et al., 2019). Indeed, we observed a decrease in IL-6 mRNA level in cells expressing MCPIP2 after induction with
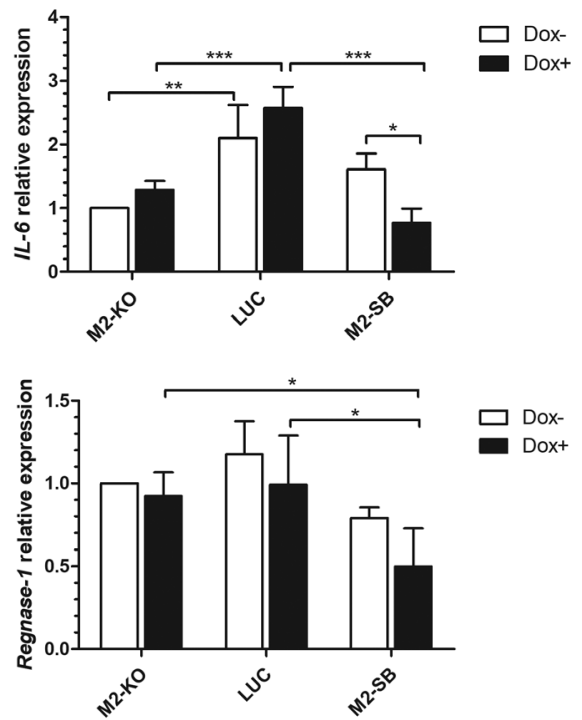

C

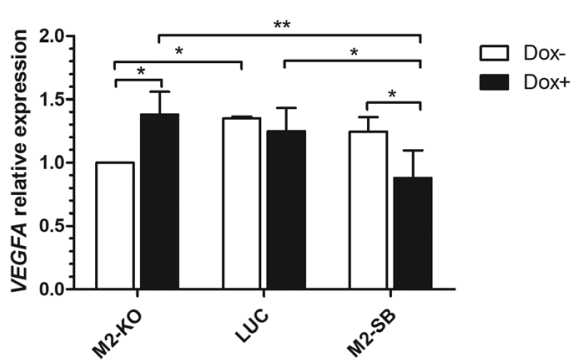

Figure 4. Influence of Sleeping Beauty system and MCPIP2 overexpression on the level of IL-6, Regnase-1 and VEGFA mRNA.

(A) IL-6 (B) Regnase-1 (C) VEGF mRNA expression profiles in U251MG-M2-KO cells (M2-KO), U251-MG-LUC (LUC) or U251-MG-M2-SB (M2-SB) with inducible overexpression of MCPIP2 or luciferase. Cells were treated with doxycycline (Dox+) for 24 hours or left untreated (Dox-) and the levels of the mRNAs of interest were measured by real-time PCR analysis. The plots show mean results from three independent experiments $(n=3) \pm$ S.D., ${ }^{*} p<0.05$, ${ }^{* *} p<0.01$, ${ }^{* * *} p<0.001$. Two-way ANOVA with Bonferroni's post-hoc test was used.

Dox (Fig. 4A, M2-SB Dox+) in comparison to the control cells expressing luciferase (Fig. 4A, LUC Dox+). A similar pattern of regulation was observed for Regnase-1 mRNA (Fig. 4B, LUC Dox+ and M2-SB Dox+). However, the level of transcripts that are not regulated by MCPIP2, such as VEGFA mRNA, is also influenced by the Dox-induced MCPIP2 overexpression (Fig. 4C, LUC Dox+ and M2-SB Dox+). Thus, the system influences gene expression in MCPIP2-independent manner. Moreover, we have also observed an increase in the level of IL-6 mRNA in the control U251-MG-LUC cells, in comparison to its level in the U251-MG-M2-KO cells (Fig. 4A). This suggests that the system itself influences changes in the level of IL- 6 mRNA. This phenomenon is not reflected by changes in the level of all investigated transcripts. The level of Regnase- 1 mRNAs is not influenced by SB modification of the U251-MG-M2-KO genome (Fig. 4B).

The observed stimulation of IL- 6 expression caused by transposon-mediated genome modification may be explained by specificity of the cell line used in our experiments. Astrocytoma is derived from astrocytes which belong to the facultative antigen-presenting cells. Following infection of the central nervous system with DNA viruses, astrocytes and microglia produce key anti-viral and inflammatory mediators, including IL-6 (Van Wag- 
oner \& Benveniste, 1999). The DNA sensors such as DAI (DNA-dependent activator of interferon-regulatory factors) and cGAS (cyclic GMP-AMP synthase) have been identified in murine glial cells (Jeffries \& Marriott, 2018). Thus, transfection of astrocytes with foreign DNA will result in a proinflammatory response. However, edition of the genome by the SB system is stable, the transcripts level was analyzed in stable-transfected cells after selection of positive/modified cells (puromycin resistance). Therefore explanation of the observed changes in IL-6 mRNA level (and lack of changes in Reg-1 and VEGFA mRNA level) in the SB-modified control cells is debatable. Taken together, our results indicate that the Sleeping Beauty approach, although very fast, efficient and versatile, may not be the best method for investigating changes in the transcriptome since it induces changes in the level of some transcripts.

\section{REFERENCES}

Chomczynski P, Sacchi N (2006) The single-step method of RNA isolation by acid guanidinium thiocyanate-phenol-chloroform extraction: Twenty-something years on. Nat. Protoc. 1: 581-585. https:// doi.org/10.1038/nprot.2006.83

Gaj T, Gersbach CA, Barbas CF 3rd (2013) ZFN, TALEN, and CRISPR/Cas-based methods for genome engineering. Trends Biotechnol. 31: 397-405. https://doi.org/10.1016/j.tibtech.2013.04.004

Gantier MP, Williams BR (2007) The response of mammalian cells to double-stranded RNA. Cytokine Growth Factor Rev. 18: 363-371. https://doi.org/10.1016/j.cytogfr.2007.06.016

Hendel A, Bak RO, Clark JT, Kennedy AB, Ryan DE, Roy S, Steinfeld I, Lunstad BD, Kaiser RJ, Alec B (2015) Chemically modified guide RNAs enhance CRISPR-Cas genome editing in human primary cells. Nat. Biotechnol. 33: 985-989. https://doi.org/10.1038/nbt.3290

Hudecek M, Izsvák Z, Johnen S, Renner M, Thumann G, Ivics Z (2017) Going non-viral: the Sleeping Beauty transposon system breaks on through to the clinical side. Crit. Rev. Biochem. Mol. Biol. 52: 355-380. https://doi.org/10.1080/10409238.2017.1304354

Jeffries AM, Marriott I (2018) Human microglia and astrocytes express cGAS-STING viral sensing components. Neurosci Lett. 29: 53-56. https://doi.org/10.1016/j.neulet.2017.08.039
Kebriaei P, Izsvák Z, Narayanavari SA, Singh H, Ivics Z (2017) Gene therapy with the Sleeping Beauty transposon system. Trends Genet. 33: 852-870. https://doi.org/10.1016/j.tig.2017.08.008

Kotterman MA, Chalberg TW, Schaffer D V. (2015) Viral vectors for gene therapy: translational and clinical outlook. Annu. Rev. Biomed. Eng. 17: 63-89. https://doi.org/10.1146/annurev-bioeng-071813-104938

Kowarz E, Löscher D, Marschalek R (2015) Optimized Sleeping Beauty transposons rapidly generate stable transgenic cell lines. Biotechnol. J. 10: 647-653. https://doi.org/10.1002/biot.201400821

Lam JKW, Chow MYT, Zhang Y, Leung SWS (2015) siRNA versus miRNA as therapeutics for gene silencing. Mol. Ther. Nucleic Acids 4: https://doi.org/10.1038/mtna.2015.23

Liu M, Rehman S, Tang X, Gu K, Fan Q, Chen D (2019) Methodologies for improving HDR efficiency. Front. Genet. 9: 691. https://doi. org/10.3389/fgene.2018.00691

Qiu P, Shandilya H, Alessio JMD, Connor KO, Durocher J, Gerard GF (2004) Mutation detection using surveyor nuclease. Biotechniques 36: 702-707. https://doi.org/10.2144/04364PF01

Ranganathan S, Sridharan M, Carte J, Ravinder N, Quintanilla R, Zou Y, Chesnut JD, Chen W, Kumar S, Potter J, Roark N, Liang X (2015) Rapid and highly efficient mammalian cell engineering via Cas9 protein transfection. J. Biotechnol. 208: 44-53. https://doi. org/10.1016/j.jbiotec.2015.04.024

Till BJ, Zerr T, Comai L, Henikoff S (2006) A protocol for TILLING and Ecotilling in plants and animals. Nat. Protoc. 1: 2465-2477. https://doi.org/10.1038/nprot.2006.329

Vavouri T, Semple JI, Garcia-Verdugo R, Lehner B (2009) Intrinsic protein disorder and interaction promiscuity are widely associated with dosage sensitivity. Cell 138: 198-208. https://doi.org/10.1016/j. cell.2009.04.029

Van Wagoner N, Benveniste E (1999) Interleukin-6 expression and regulation in astrocytes. J. Neuroimmunol. 100: 124-139. https://doi. org/10.1016/S0165-5728(99)00187-3

Walther W, Stein U (2000) Viral vectors for gene transfer: a review of their use in the treatment of human diseases. Drugs 60: 249-71. https://doi.org/10.2165/00003495-200060020-00002

Wawro M, Wawro K, Kochan J, Solecka A, Sowinska W, LichawskaCiesla RA, Jura J, Kasza A (2019) ZC3H12B/MCPIP2, a new active member of the ZC3H12 family. RNA 25: 840-856. https:// doi.org/10.1261/rna.071381.119

Westermark B, Ponten J, Hugosson R (1973) Determinants for the establishment of permanent tissue culture lines from human gliomas. Acta Pathol. Microbiol. Scand. 81: 791-805. https://doi. org/10.1111/j.1699-0463.1973.tb03573.x

Zucca S, Pasotti L, Politi N, Gabriella M, Angelis C De, Magni P (2013) A standard vector for the chromosomal integration and characterization of BioBrick TM parts in Escherichia coli. J. Biol. Engenering 7: 1-13. https://doi.org/10.1186/1754-1611-7-12 